\title{
CLINICAL AND LABORATORY CHARACTERISTICS OF PATIENTS AFTER SURGERY OF TIBIAL FRACTURE AT SOC TRANG PROVINCIAL GENERAL HOSPITAL
}

\author{
Tran Quoc Tuan', Nguyen Minh Hiep ${ }^{2}$ \\ ${ }^{1}$ Soc Trang Province General Hospital \\ ${ }^{2}$ Phuong Chau International Hospital Can Tho
}

\begin{abstract}
Purpose: Examine clinical and laboratory characteristics of patients after surgery of tibial fracture at Soc Trang Province General Hospital. Participants and methods: Patients were prescribed to tibial fracture surgery at Orthopedic Surgery Department - Soc Trang Provincial General Hospital from November 1, 2020 to April 30, 2021. Methods of crosssectional descriptive study on 186 patients. Results: The mean age of the participants was 45.11 \pm 17.44 (min-max:15-89); the majority were men, accounting for $62.4 \%$. After surgery, the indicators of blood pressure, pulse, breathing rate improved. $66.7 \%$ of participants had pain after surgery. Most of the participants had normal lab test results after surgery. Most of the participants had X-rays, showing that $99.5 \%$ had good surgery results. The patients had ultrasound (17.7\%), CT scan (19.4\%); cultured bacteria (5.9\%), postoperative infectious complications (0.5\%); loss of sensation (45.2\%); compressed the cavity (0). Conclusion: After surgery, patients often had pain symptoms and the postoperative infection rate was as low as $0.5 \%$.
\end{abstract}

Keywords: clinical and laboratory characteristics, tibial fracture surgery, Soc Trang.

\section{INTRODUCTION}

Tibial fracture is a common trauma emergency at provincial hospitals, currently accounting for $>15 \%$ [1]. Common causes are traffic, occupational and daily life accidents, which make the Department of Orthopedic Surgery overloaded. However, this is a type of fracture that is difficult to correct, leaving various permanent sequelae. Symptoms such as joint pain in the legs, limitations in work and life, misalignment, osteoarthritis, stiffness, etc., should be less and less conservative treatment, only applicable to non-displaced fractures. The scientific advances in instrumentation, infection control, and increasingly advanced technical expertise, the indications for surgical treatment of osteosarcoma have been expanded that is appropriate to each case's characteristics, bringing more effective results. Nursing care is very important to ensure the patients's recover after surgery, however, it is necessary to optimize the patient's recorded characteristics in terms of clinical and laboratory characteristics, then providing the most comprehensive treatment and care plan, helping the patient recover function after surgery as soon as possible.
Cor. author: Tran Quoc Tuan

Address: Soc Trang Province General Hospital

Email: tuantutun5@gmail.com
Received: Dec 20, 2021

Accepted: Feb 23, 2022

Published: Feb 24, 2022 
Therefore, the researcher conducted the study with the goal of examining the clinical and laboratory characteristics of patients after tibial fracture surgery at Soc Trang Provincial General Hospital.

\section{PARTICIPANTS AND METHODS}

2.1. Participants: The patient was prescribed to tibial fracture surgery at the Department of Orthopedic Surgery, Soc Trang Province General Hospital.

Inclusion criteria: Patient aged 15 years and over; tibial fracture surgery (Vis, splints, intramedullary nails); Patients were able to communicate and fully documented in medical records;.

Exclusion criteria: Patient had pathological fracture, multiple severe trauma.

\subsection{Setting}

The research was conducted from November 1, 2020 to April 30, 2021 at the Orthopedic Surgery Department, Soc Trang Province General Hospital

\subsection{Research design}

Cross-sectional descriptive study

\subsection{Sample and sampling}

- Sample size was counted applying the formula for calculating sample size to estimate the population proportion in the study

$$
n=Z_{1-\alpha / 2}^{2} \frac{p(1-p)}{d^{2}}
$$

$\mathrm{n}$ : The appropriate sample size with probability $\alpha=0.05$ with $Z 1-\alpha / 2=1.96 ; p$ : Estimating the rate of good care outcomes in patients after fracture surgery (Nguyen Huu Ngoc with good and very good results accounted for $78.12 \%$, with $p=0.7812$ [2]); $\mathrm{d}$ : margin of error, $d=0.06$. Substituting into the formula, the study sample size was 183, Finally, the researcher collected 186 patients.

- Sampling method was the purposive sampling.

2.5. Variables, indicators, evaluation criteria: The patient characteristics upon admission, intraoperative characteristics, laboratory characteristics, treatment outcomes and postoperative complications.

Criteria for assessing postoperative pain in the study, researcher used part one of the Numeric Rating Scale (NRS) consisting of 4 questions asking patients to self-report their pain score at 4 time points while the patient feels "most painful", "least painful", "moderate pain" and "current pain". Each question used a scale of 0 to 10 to rate, 0 was no pain and 10 was a lot of pain [3].

\subsection{Data collection}

The researcher interviewed and examined the patients and reviewed medical records

\subsection{Data analysis}

Data was entered and cleaned by Epidata 3.1 software. Data analysis using STATA 16.0 software. Data was analyzed by descriptive statistics using percentage, mean value.

\subsection{Ethic consideration}

The study was approved by the Institutional Review Board of Thang Long University and approved by leaders of Soc Trang Province General Hospital. All patient information was guaranteed to be kept confidential. The data was only provided for research. 


\section{RESULTS}
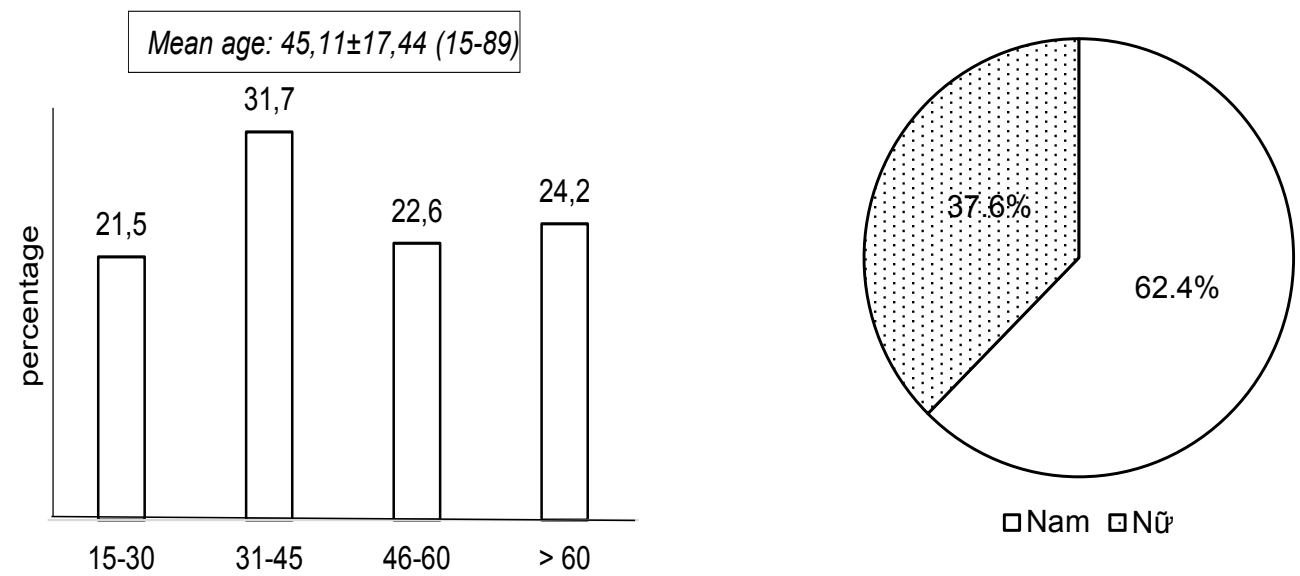

Figure 1. Distribution of participants by age, sex

The average age of the participants was $45.11 \pm 17.44$ (min-max:15-89). The proportion of participants aged $31-45$ years old, accounted for the highest $31.7 \%$. The majority was men, accounting for $62.4 \%$.

Table 1. Vital signs after surgery

\begin{tabular}{|c|c|c|c|c|c|c|}
\hline \multicolumn{2}{|c|}{ Variables } & \multicolumn{2}{|c|}{ Before surgery } & \multicolumn{2}{|c|}{ After surgery } & \multirow{2}{*}{$\mathbf{p}$} \\
\hline & & $\mathrm{n}$ & $\%$ & $\mathrm{n}$ & $\%$ & \\
\hline \multirow{2}{*}{$\begin{array}{l}\text { Blood pressure } \\
(\mathrm{mmHg})\end{array}$} & High & 18 & 9,7 & 1 & 0,5 & \multirow{2}{*}{$<0,01$} \\
\hline & Normal & 168 & 90,3 & 185 & 99,5 & \\
\hline \multirow{3}{*}{$\begin{array}{c}\text { Pulse } \\
\text { (beats/minute) }\end{array}$} & Fast & 8 & 4,3 & 6 & 3,3 & \multirow{3}{*}{0,89} \\
\hline & Normal & 177 & 95,2 & 179 & 96,2 & \\
\hline & Slow & 1 & 0,5 & 1 & 0,5 & \\
\hline \multirow{3}{*}{$\begin{array}{c}\text { Breathing } \\
\text { (beats/minute) }\end{array}$} & Fast & 35 & 18,8 & 20 & 10,7 & \multirow{3}{*}{0,06} \\
\hline & Normal & 147 & 79,0 & 164 & 88,2 & \\
\hline & Slow & 4 & 2,2 & 2 & 1,1 & \\
\hline \multicolumn{2}{|c|}{ Total } & 186 & 100 & 186 & 100 & \\
\hline
\end{tabular}

All participants improved indicators of blood pressure, pulse, and breathing rate after surgery; in which the rate of high blood pressure decreased after surgery from $9.7 \%$ to $0.5 \%$; The abnormal breathing rate decreased from $21 \%$ before surgery to $11.8 \%$ after 
surgery, the difference was statistically significant with $p<0.05$; rate of participants with abnormal pulse before surgery was $4.8 \%$, the rate decreased to $3.8 \%$ after surgery; However, the difference was not statistically significant with $p>0.05$.

Table 2. Post-operative pain level (Numeric Rating Scale - NRS)

\begin{tabular}{|ccc|}
\hline Pain level & Number (n) & Rate (\%) \\
\hline No pain & 62 & 33,3 \\
\hline Less pain & 33 & 17,7 \\
\hline Moderate pain & 90 & 48,4 \\
\hline Severe and intense pain & 1 & 0,5 \\
\hline Total & $\mathbf{1 8 6}$ & 100 \\
\hline
\end{tabular}

Rate of participants having pain after surgery was $66.7 \%$; in which, rate of severe and severe pain, moderate pain and less pain were $0.5 \%, 48.4 \%, 17.7 \%$, respectively.

Table 3. Post-operative laboratory characteristics

\begin{tabular}{|c|c|c|c|}
\hline \multicolumn{2}{|c|}{ Laboratory characteristics } & Number (n) & Rate (\%) \\
\hline \multirow{3}{*}{ RBC } & Normal & 151 & 81,2 \\
\hline & Abnormal & 35 & 18,8 \\
\hline & $\bar{X} \pm S D(\min -\max )$ & $4,45 \pm 0,83$ & $3,05-9,40$ \\
\hline \multirow{3}{*}{ WBC } & Normal & 166 & 89,3 \\
\hline & Abnormal & 20 & 10,7 \\
\hline & $\bar{X} \pm S D$ & $9,11 \pm 1,98$ & $2,94-15,1$ \\
\hline \multirow{3}{*}{$\mathrm{HgB}$} & Normal & 185 & 99,5 \\
\hline & Abnormal & 1 & 0,5 \\
\hline & $\bar{X} \pm S D$ & $12,60 \pm 1,68$ & $4,49-17,80$ \\
\hline \multirow{3}{*}{ Hct } & Normal & 178 & 95,7 \\
\hline & Abnormal & 8 & 4,3 \\
\hline & $\bar{X} \pm S D$ & $37,72 \pm 5,00$ & $13,50-51,80$ \\
\hline \multirow{3}{*}{ PLT } & Normal & 179 & 96,2 \\
\hline & Abnormal & 7 & 3,8 \\
\hline & $\bar{X} \pm S D$ & $242,02 \pm 67,22$ & $40-517$ \\
\hline
\end{tabular}




\begin{tabular}{|cccc|}
\hline Laboratory characteristics & Number $(\mathbf{n})$ & Rate (\%) \\
\hline PT & Normal & 186 & 100 \\
& Abnormal & 0 & 0 \\
& $\bar{X} \pm$ SD & $90,68 \pm 18,51$ & $5-130$ \\
APTT & Normal & 182 & 97,8 \\
& Abnormal & 4 & 2,2 \\
& $\bar{X} \pm S D$ & $28,15 \pm 3,87$ & $11,0-44,60$ \\
\hline Ure & Normal & 182 & 97,9 \\
& Abnormal & 4 & 2,1 \\
& $\bar{X} \pm S D$ & $4,71 \pm 1,30$ & $1,70-9,40$ \\
\hline Creatinin & Normal & 183 & 98,4 \\
& Abnormal & 3 & 1,6 \\
Blood & $\bar{X} \pm S D$ & $74,321 \pm 14,49$ & $33-124$ \\
\hline glucose & Normal & 153 & 82,3 \\
& Abnormal & 33 & 17,7 \\
& $\bar{X} \pm S D$ & $6,20 \pm 1,67$ & $2,80-11,0$ \\
\hline
\end{tabular}

Most of the participants after surgery, the blood counts were in the normal range. The percentage of participants with abnormal indexes was not high, of which the participants had abnormal results of red blood cells (18.8\% of); white blood cells $(10.7 \%), \mathrm{HgB}(0.5 \%)$; 4.3\% (HCT); platelets (3.8\%); APTT (2.2\%), none of the participants had abnormality in PT

The proportion of participants had abnormal results of urea index (2.1\%); Creatinine (1.9\%); however, $17.7 \%$ of participants had abnormal blood glucose.

Table 4. X-ray and ultrasound results of patients after surgery

\begin{tabular}{|c|c|c|c|}
\hline \multicolumn{2}{|c|}{$X Q$, ultrasound } & \multirow{2}{*}{$\begin{array}{c}\text { Number (n) } \\
185\end{array}$} & \multirow{2}{*}{$\begin{array}{c}\text { Rate \% } \\
99,5\end{array}$} \\
\hline YO & Good & & \\
\hline$\lambda Q$ & Not good & 1 & 0,5 \\
\hline \multirow{2}{*}{ Ultrasound } & Yes & 33 & 17,7 \\
\hline & No & 153 & 82,3 \\
\hline \multirow{2}{*}{ CT-Scaner } & Yes & 36 & 19,4 \\
\hline & No & 150 & 80,6 \\
\hline \multirow{2}{*}{ Bacterial culture } & Yes & 11 & 5,9 \\
\hline & No & 175 & 94,1 \\
\hline \multicolumn{2}{|c|}{ Total } & 186 & 100 \\
\hline
\end{tabular}


Most of the participants had X-rays indicating that $99.5 \%$ had good surgery outcomes. The participants had ultrasound (17.7\%), CT scan (19.4\%); cultured bacteria (5.9\%).

Table 5. Postoperative complications

\begin{tabular}{|ccc|}
\hline Symptoms & Number $(\mathbf{n})$ & Rate $\%$ \\
\hline Post-operative infection & 1 & 0,5 \\
\hline Leg numbness & 84 & 45,2 \\
\hline CEK & 0 & 0 \\
\hline Total & 186 & 100 \\
\hline
\end{tabular}

After treatment, $100 \%$ of patients were discharged from the hospital, however, there were 1 per 186 participants with postoperative infection $(0.5 \%)$, leg numbness $(45.2 \%)$, CEK (0).

Table 6. Average duration of treatment and post-surgery

\begin{tabular}{|c|c|c|}
\hline Time & Mean \pm SD & Min - max \\
\hline Treatment time (days) & $12,46 \pm 4,65$ & $7-39$ \\
\hline Post-sugery time (days) & $8,99 \pm 4,32$ & $3-34$ \\
\hline
\end{tabular}

The mean hospital stay was $12.46 \pm 4.65$ days, the shortest hospital stay was 7 days, and the longest was 39 days. The average time after surgery was $8.99 \pm 4.32$, the shortest time after surgery was 3 days, the longest time after surgery was 34 days.

\section{DISCUSSION}

The study was carried out on 186 tibial fracture surgery patients at Orthopedic Surgery Department, Soc Trang Province General Hospital. In which, the average age of the study participants was $45.11 \pm 17.44$ (min-max:15-89); the majority were men, accounting for $62.4 \%$, main occupation was farmer $(61.3 \%)$, pensioner (25.8\%).

Most of the patients with respiratory rate not within the normal range, slow breathing pattern, apnea or shallow tachypnea as signs of respiratory failure. In the study, all participants improved indicators of blood pressure, pulse, and breathing rate after surgery. Research by Nguyen Gia Dung (2019) [4] at the first 24 hours after surgery, there were 13 patients with bleeding at the incision site $(11.2 \%), 6$ patients with low or high body temperature (5.2\%) ), 12 patients with tachycardia (10.3\%), 2 patients with tachypnea $(1.7 \%), 11$ patients with high blood pressure $(9.5 \%)$. Pham Van Kia's study recorded two cases of abnormal temperature accounting for $5.4 \%$ and one patient with abnormal blood pressure accounting for $2.7 \%$ [1]. 
Postoperative pain depends on psychophysiology, patient tolerance, nature of surgery, degree of surgical trauma. Our results showed that the rate of participants having pain after surgery was $66.7 \%$; rate of severe and intense pain was $0.5 \%$; rate of moderate pain was $48.4 \%$; rate of pain less was $17.7 \%$. Research by Nguyen Gia Dung (2019) [4] at the first 24 hours after surgery, most patients had moderate pain after surgery, accounting for 90.5\%, 5 patients had severe and intense pain. In Pham Van Kia's study, there were 16 very painful patients, accounting for $43.2 \%$ [1].

Postoperative complications were examined. There were 1 per 186 participants with postoperative infection $(0.5 \%)$; leg numbness (45.2\%), CEK (0). There was a participants having postoperative infection with open fractures. When an open fracture occurs, the tip of the broken bone may be exposed at the wound or not, but only indirectly, with blood and marrow fat exuding from the wound. Vijay Karande's study recorded 6 patients with superficial infections and of which 4 participants had uncontrolled diabetes [5]. Infection status was related to degree of soft tissue and bone damage, degree of wound contamination, first aid and early antibiotic use, time of management and quality of resection. Most of the participants after surgery had the blood count results in the normal range, the percentage of participants with abnormal indexes was not high. Research by Nguyen Gia Dung (2019) [4] the majority of patients also had post-operative lab indicators within normal limits; the proportion of participants with abnormal test results were urea index $(2.3 \%)$; Creatinine $(1.9 \%)$; however, blood glucose $(17.9 \%)$.
Most participants took X-ray 99.5\%; ultrasound (17.7\%), CT scan (19.4\%); cultured bacteria (5.9\%). In the study of Dao Thien Tien (2012) [6] Postoperative X-ray showed that 43 per 47 patients $(91.5 \%)$ were orthopedic straight axis fractures by Kuntscher intramedullary nail fusion.

\section{CONCLUSION}

The average age of the study group was 45.11 \pm 17.44 (min-max:15-89); the majority was men, accounting for $62.4 \%$. After surgery, the indicators of blood pressure, pulse, breathing rate improved. $66.7 \%$ pain was occured after surgery. Most of the patients had normal paraclinical index after surgery. Most of the participants had X-rays, showing that $99.5 \%$ had good correction results. Participants had ultrasound (17.7\%), CT scan (19.4\%); cultured bacteria (5.9\%), postoperative infectious complications $(0.5 \%)$; loss of sensation $(45.2 \%)$; There were no patients with CEK.

\section{REFERENCES}

1. Pham Van Kia and Tra Thi Hoang Yen (2020), Evaluation of the results of post-operative care combined with broken bones of the tibial bone at the Department of Surgery - Neurology, Bong Son Regional General Hospital from March 2019 to October 2020, Basic project, Department of Health of Binh Dinh province.

2. Nguyen Huu Ngoc (2003), "Evaluation of treatment results for fractures of the medial ankle, lower third of the fibula by fusion surgery", Vietnam Medical Journal, Special Issue T10-2003, p. 207-212. 
3. Craig $T$ Hartrick, Juliann $P$ Kovan và Sharon Shapiro (2003), "The numeric rating scale for clinical pain measurement: a ratio measure?", Pain Practice, 3(4), p. 310-316. https://doi.org/10.1111/j.15307085.2003.03034.x

4. Nguyen Gia Dung (2019), Characteristics, care of patients with lower extremity fracture surgery and factors related to care at the Department of Surgery, Duc Giang hospital, Master of Nursing Thesis, Thang Long University.
5. Vijay Karande, Vivek $P$ Nikumbha, Ashok Desai et al. (2017), "Study of surgical management of malleolar fractures of ankle in adults", International Journal of Orthopaedics, 3(3), p. 783-787. https://doi. org/10.22271/ortho.2017.v3.i3k.118

6. Dao Thien Tien (2012), "Evaluating the results of treatment of first- and thirddegree open fractures between 2 shin bones by KŨNTSCHER intramedullary nailing method", Journal of Military Medicine, 4-2012, p. 1-8. 